\title{
Correction of post ankylotic facial asymmetry with bimaxillary distraction osteogenesis-Case report
}

\author{
U. S. Pal, Nimisha Singh, Laxman R. Malkunje, R. K. Singh, Chandan Gupta, Sharad Chand
}

Department of Oral and Maxillofacial Surgery, C. S. M. Medical University, Lucknow, India

Email: uspalkgmc@yahoo.co.in

Received 23 August 2012; revised 23 September 2012; accepted 3 October 2012

\begin{abstract}
Facial asymmetry can be acquired or congenital. Patients with facial asymmetry are not always functionally disturbed by the malfunction but are usually very much disturbed by their external appearance. Depending on the degree of asymmetry and deformation, the surgical procedure may vary in complexity and extent. The extent of surgery can range from a genioplasty procedure to bimaxillary osteotomy, concomitant with augmentation surgery, genioplasty and craniofacial implants along with mandibular distraction. In severe cases, the soft tissue structures on the affected side may constitute an incredible resistance to stretching and can make the surgery considerably more difficult and liable to relapse. Here we represent a case of post ankylotic facial asymmetry with occlusal cant which was treated by bimaxillary distraction osteogenesis. Simultaneous mandibular and maxillary distraction corrected the facial asymmetry without disturbing the pre-existing compensated dental occlusion, and so there was no need for prolonged and difficult orthodontic treatment.
\end{abstract}

Keywords: Facial Asymmetry; Occlusal Cant; Bimaxillary Distraction Osteogenesis

\section{INTRODUCTION}

Adult patients with TMJ ankylosis usually have various degrees of anatomical facial deformities, including microgenia, reduced lower facial height, poor jaw neck definition and of occlusal discrepancy. In unilateral patients, facial asymmetry is less associated with occlusal discrepancy; however, canting of their occlusal plane is more due to mandibular hypoplasia on the affected side, with secondary epsilateral vertical deficiency in the maxillary process $[1,2]$.

The correction of facial deformities following the treatment of TMJ ankylosis remains a difficult and challenging problem in oral and maxillofacial surgery. Vari- ous techniques for treating this have been described but no uniform protocol exists with successful results. Expected complications may vary from limited inter incisal opening due to relapse, loss of vertical height of the affected ramus, foreign body reactions and re-ankylosis [3]. The treatment of TMJ ankylosis requires restoration of proper mandibular form, length and vertical dimension, occlusal stability and satisfactory joint movement.

Distraction osteogenesis has recently become a main stay for the treatment of craniofacial syndromes with mandibular hypoplasia including TMJ ankylosis. Its success in lengthening the mandible opens new perspectives for interceptive therapy, where other surgical techniques including orthognathic surgery and/or bone grafting procedures have not proved to be satisfactory $[4,5]$.

Many authors [6,7] have reported marked occlusal disturbances following mandibular distraction osteogenesis which are sometimes difficult to be corrected orthodontically. When treating patients with TMJ ankylosis, some authors (Lopez and Doglitti) [8] prefer to first restore the jaw movements, and address the secondary facial deformities afterwards. Ortiz Monasterio et al. [9] and Cho et al. [10] have recommended simultaneous bimaxillary distraction osteogenesis with the use of external devices in patients with hemifacial microsomia for correction of their facial asymmetry. Similarly, many others, including Guerrero et al. [11], Papageorge and Apostolidis [12] and Liang et al. [4], prefer simultaneous mandibular distraction and arthroplasty in patients with TMJ ankylosis and mandibular hypoplasia. An unstable proximal condylar segment remains a problem during the distraction process. In this case the interpositional arthroplasy was done 2 years back and we did bimaxillary distraction osteogenesis to correct the facial asymmetry and occlusal cant.

\section{CASE REPORT}

Under general anaesthesia with nasotracheal intubation, $2 \%$ lidocaine mixed with $1: 100,000$ epinephrine was infiltrated. An incision was made along the upper buccal sulcus, $7 \mathrm{~mm}$ from the mucogingival junction. Using 
subperiosteal dissection, the anterior and lateral aspects of the maxilla were exposed to the level of the pterygomaxillary junction.

The nasal spine was exposed and the subperiosteal undermining was extended along the floor, the inferior portion of the septum and the lateral walls of the nose. The osteotomy line was determined $5 \mathrm{~mm}$ above the roots of the teeth. With a reciprocating saw, a complete horizontal osteotomy was made between the piriform aperture and the maxillary tuberosity. The nasal septum was completely freed from the vomer, and pterygomaxillary junction of affected side was freed using a curved Tessier osteotome. Rowe forceps were used for the downfracture of the maxillary segment. After LeFort I osteotomy, intermaxillary fixation was performed to obtain a stable occlusal relationship. A second incision was made along the inferior buccal sulcus on the affected side at the level of the ascending ramus. Using subperiosteal dissection, both sides of the ramus were exposed.

The osteotomy line was obliquely extended from the posterior part of the last molar tooth to the gonial angle. Using a side-cutting burr, a corticotomy was performed on the medial and lateral aspects of the mandible until the cancellous layer was exposed, then a wide osteotome was inserted and twisted to achieve a greenstick fracture of the mandible. The continuity of the inferior alveolar neurovascular bundle was preserved. An internal distraction device was placed and fixed with $5 \mathrm{~mm}$ screws.

Distraction was initiated 5 days after the operation at a rate of $1 \mathrm{~mm}$ per day. Before the start of distraction, IMF was done to make the maxillary and mandibular segment a single unit for simultaneous distraction of maxilla and mandible. The distraction process was continued until the planned elongation was complete, both oral commissures were symmetrical and the lower inter incisor notch had moved slightly towards the unaffected side compared to the upper interincisor notch. After completion of distraction, plating at the affected side buttress region was carried out under Local Anaesthesia and then IMF was released. The distraction device was left in place for 6 - 8 weeks until we observed radiological evidence of new mandibular bone formation.

Preoperatively, the deviation of the occlusal plane from the horizontal plane (occlusion cant) was $17 \mathrm{~mm}$ (Figures 1-4).

Preoperative cephalometric values are:

SNA angle-83 degree;

SNB angle -70 degree;

ANB angle-13 degree.

The mandibular elongation of $17 \mathrm{~mm}$ was done. Preoperatively, the vertical dimension of the maxilla from the infraorbital rim to the occlusal plane was $51 \mathrm{~mm}$. After distraction, the vertical dimension $53 \mathrm{~mm}$ (Figures 5-8).

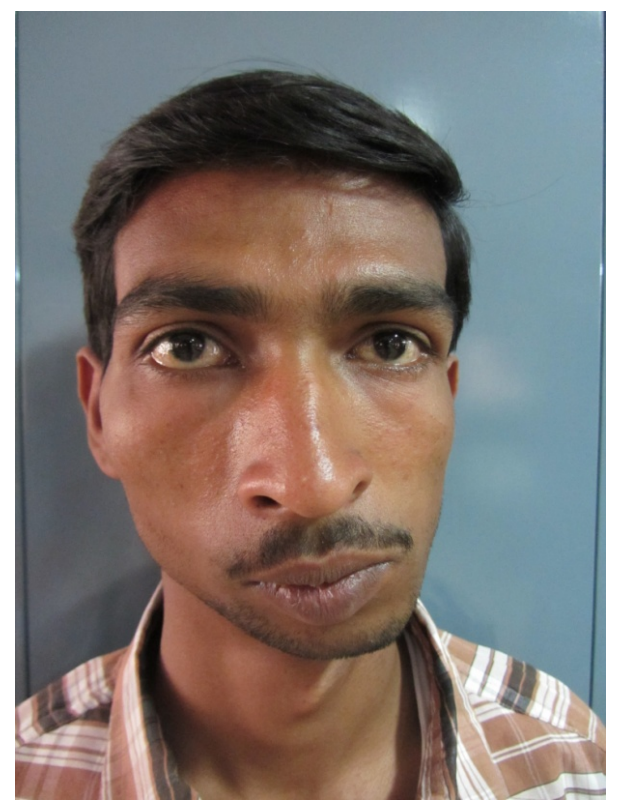

Figure 1. Pre operative photograph of patient.

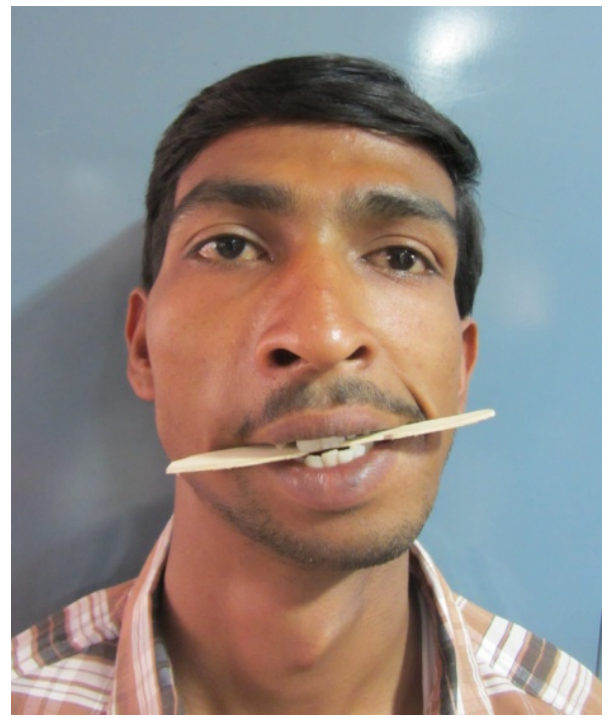

Figure 2. Pre operative photograph of patient showing occlusal canting.

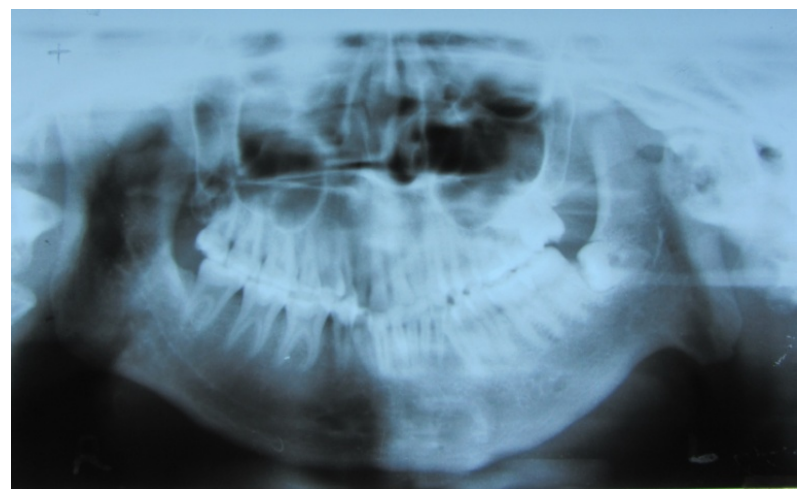

Figure 3. Pre operative orthopantomogram (OPG). 


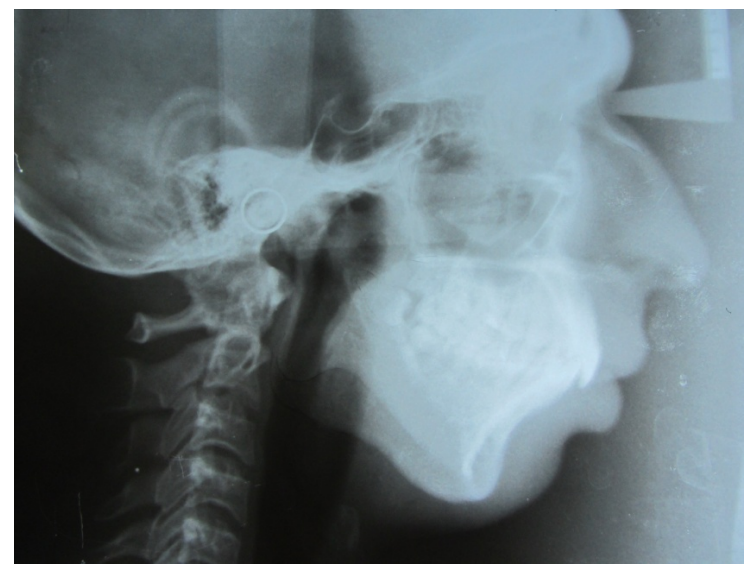

Figure 4. Pre operative lateral cephalogram.

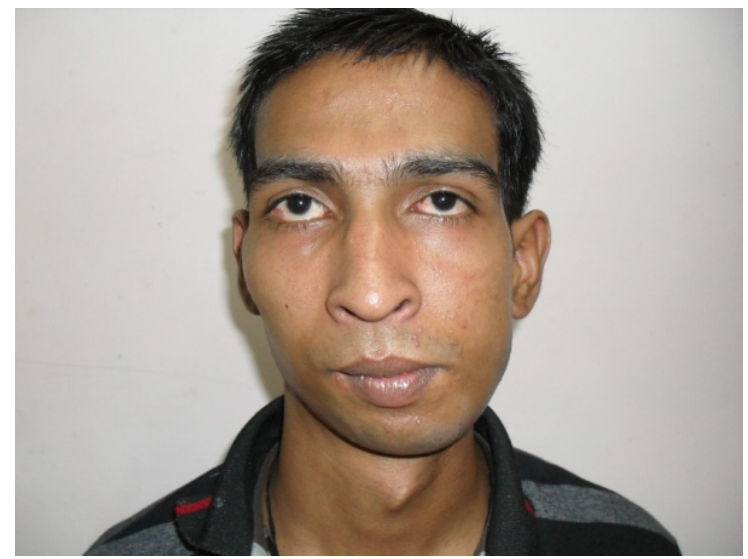

Figure 5. Post operative photograph of patient.

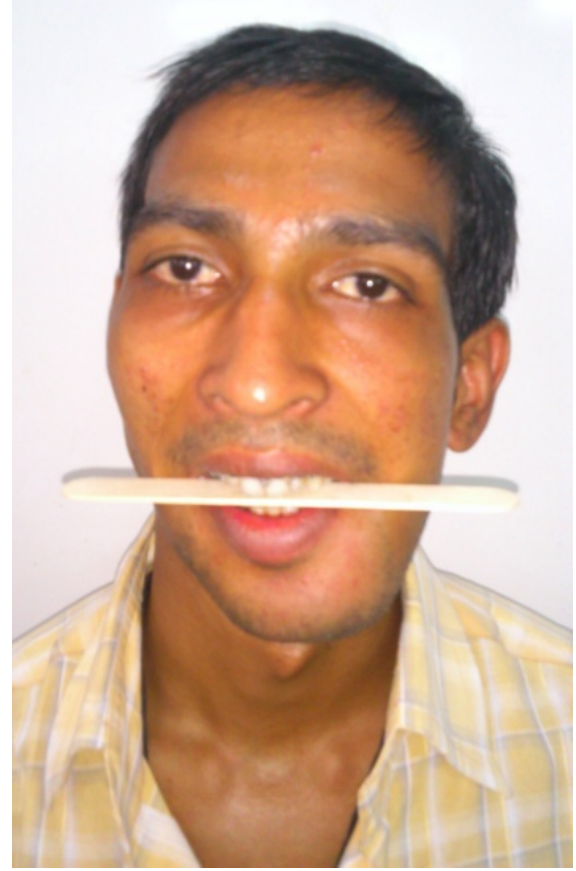

Figure 6. Post operative photograph of patient showing corrected occlusal canting.

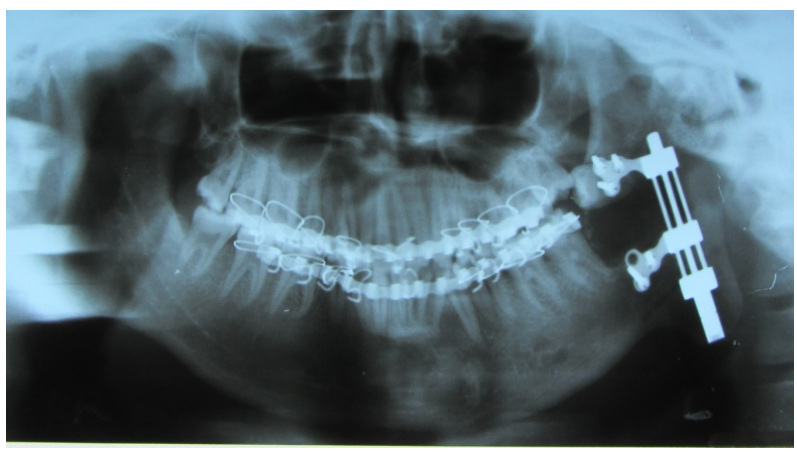

Figure 7. Orthopantomogram (OPG) after completion of distraction.

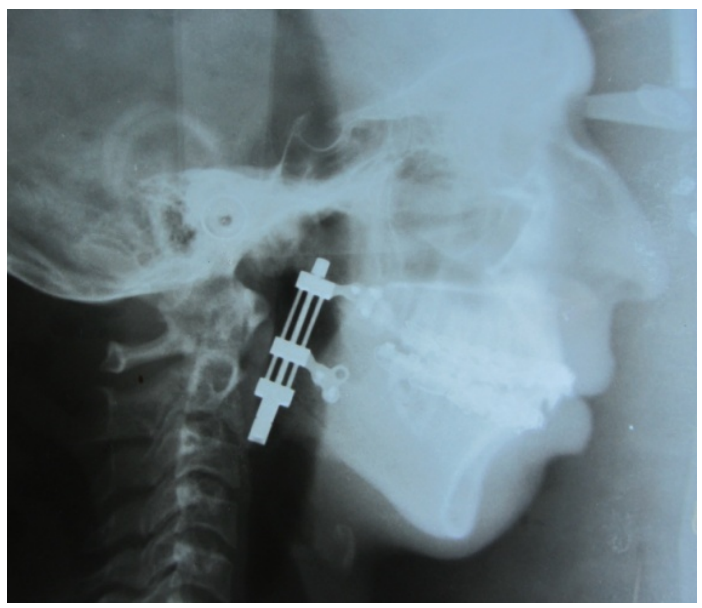

Figure 8. Post operative lateral cephalogram.

Postoperative cephalometric values are:

SNA angle-82 degree;

SNB angle-72 degree;

ANB angle-10 degree.

To assess any relapse, the cephalometry 6 months to 1 year after distraction was compared with that immediately after the removal of the distraction devices.

However, the change in the external appearance was minimal, so no further procedures were performed. Mild difficulties with mastication and a limited mobility of the temporomandibular joint were experienced due to the long period of fixation, however, the movement in the joint improved over time.

\section{DISCUSSION}

Various studies [13-15] have reported the aetiology of temporomandibular joint (TMJ) ankylosis to be most commonly associated with trauma (13\% - 100\%), local or systemic infection ( $0 \%-53 \%)$ and systemic diseases, such as ankylosing spondylitis, rheumatoid arthritis and psoriasis (28\%), and to occur after TMJ surgery. This syndrome not only prevents mouth opening and chewing, but affects the growth and position of the mandible. This 
can eventually produce progressive facial distortion, with devastating psychosocial effects compounding the already difficult problem of not being able to open the mouth.

In the field of osteodistraction of the craniofacial skeleton, McCarthy et al. introduced unilateral mandibular elongation in patients with hemifacial microsomia or mandibular hypoplasia [16]. Molina and Ortiz Monasterio reported a series of cases of mandibular elongation using the corticotomy of the external surface of the mandible and an external distraction device [17]. Recently, Cohen introduced an intraoral distraction device to avoid external scarring [18]. Excellent aesthetic results have been obtained with the progressive elongation of hypoplastic hemimandibles because the soft tissue attached to the bone is expanded simultaneously. Other advantages of osteodistraction include the lack of donor-site morbidity and the fact that the technique is minimally invasive when compared to a free bone graft or a vascularised bone graft. For this reason, osteodistraction has been widely accepted.

Mandibular distraction in children with hemifacial microsomia produces inevitable changes in dental occlusion; however, these changes can be corrected orthodontically because of the rapid vertical growth of the maxilla in children when it is released from the restrictive effect of the mandible and soft tissues. In adults it is different, as the dental occlusion can be changed drastically by distraction osteogenesis, which requires unacceptably prolonged and difficult orthodontic treatment [9]. To avoid this problem, Ortiz-Monasterio et al. introduced the technique of simultaneous bimaxillary distraction osteogenesis using an incomplete Le Fort I maxillary osteotomy, and mandibular corticotomy to maintain a stable dental occlusion [9].

Bimaxillary distraction is indicated in patients with facial asymmetry and a canted occlusal plane. Simultaneous mandibular and maxillary distraction will correct the facial asymmetry without disturbing the pre-existing compensated dental occlusion, and so save the patient the need for prolonged and difficult orthodontic treatment $[6$, $10]$.

We used bone lengthening by gradual distraction osteogenesis as a method of filling the gap that resulted from gradual advancement of bone using the natural healing process based on the physiological rules of bone healing [19]. This approach allows the surgeon to lengthen the maxillomandibular complex simultaneously. Symmetrical skeletal appearance was also improved. Soft tissue was lengthened, which improved its appearance and reduced the extent of skeletal relapse. We left the pterygomaxillary junction of the unaffected side intact, to act as a pivot point and improve the stability of the midface as explained by Ortiz-Monasterio et al. [9].
Hemifacial microsomia is reported to be the facial asymmetry most widely treated using this technique [6, 9]. We found that facial asymmetry secondary to unilateral ankylosis of the TMJ was treated successfully in this way.

We have mentioned a case of post ankylotic facial asymmetry with occlusal cant which was treated by lengthening the ramus and leveling the occlusal plane with bimaxillary distraction osteogenesis simultaneously. We opted for this procedure because our patients were adults and had completed the growth spurts period. The total period of IMF kept was four weeks. Plating at the affected side buttress region was carried out under Local Anaesthesia and then IMF was released. This eliminated the need of keeping the patients under IMF for a prolonged period of time. This treatment gives better long term stability and soft tissue lengthening with reduced period of IMF and less discomfort to the patient, better nutritional support with minimal complications.

\section{REFERENCES}

[1] Obwegeser, H.L. (1988) Variations of a standard approach for correction of the bird-face deformity. Journal of Cranio-Maxillofacial Surgery, 16, 247-265. doi:10.1016/S1010-5182(88)80059-3

[2] Perrott, D.H., Umeda, H. and Kaban, L.B. (1994) Costochondral graft construction/reconstruction of the ramus/ condyle unit: Long-term follow-up. International Journal of Oral and Maxillofacial Surgery, 23, 321-328. doi:10.1016/S0901-5027(05)80046-3

[3] Valentini, V., Vetrano, S., Agrillo, A., Torroni, A., Fabiani, F. and Iannetti, G. (2002) Surgical treatment of TMJ ankylosis: Our experience (60 cases). Journal of Cranio-Maxillofacial Surgery, 13, 59-67.

[4] Liang, C., Wang, X., Yi, B., Li, Z., Wang, X. and Chen, B. (2002) Distraction osteogenesis for treatment of temporomandibular joint ankylosis. Chinese Medical Journal, 25, 807-809.

[5] Sadakah, A. (1997) Distraction osteogenesis of previously grafted sites in the lower jaw. Cairo Dental Journal, 13, 9-16.

[6] Padwa, B.L., Kearns, G.J., Todd, R., Troulis, M., Mulliken, J.B. and Kaban, L.B. (1999) Simultaneous maxillary and mandibular distraction osteogenesis with a semiburied device. International Journal of Oral \& Maxillofacial Surgery, 28, 2-8. doi:10.1034/j.1399-0020.1999.280102.x

[7] Politis, C., Fossion, E. and Bossuyt, M. (1987) The use of costochondral grafts in arthroplasty of the tempromandibular joint. Journal of Cranio-Maxillofacial Surgery, 15, 345-354. doi:10.1016/S1010-5182(87)80081-1

[8] Lopez, E.N. and Dogliotti, P.L. (2004) Treatment of temporomandibular joint ankylosis in children: Is it necessary to perform mandibular distraction simultaneously? Journal of Craniofacial Surgery, 15, 879-885.

[9] Ortiz-Monasterio, F., Molina, F., Andrade, L., Rodriguez, 
C. and Sainz Arregui, J. (1997) Simultaneous mandibular and maxillary distraction in hemifacial microsomia in adults: Avoiding occlusal disasters. Plastic and Reconstructive Surgery, 100, 852-861. doi:10.1097/00006534-199709001-00005

[10] Cho, B.C., Shin, D.P., Park, J.W. and Baik, B.S. (2001) Bimaxillary osteodistraction for the treatment of facial asymmetry in adults. British Journal of Plastic Surgery, 54, 491-498. doi:10.1054/bjps.2001.3629

[11] Guerrero, C.A., Pasteur, B.S. and Bell, W.H. (1997) Combined temporo-mandibular joint ankylosis release with mandibular lengthening via distraction osteogenesis. International Congress on Cranial and Facial Bone Distraction Processes, Medimond International Proceedings, Bologna.

[12] Papageorge, M.B. and Apostolidis, C. (1999) Simultaneous mandibular distraction and arthroplasty in a patient with temporomandibular joint ankylosis and mandibular hypoplasia. Journal of Oral and Maxillofacial Surgery, 57, 328-333. doi:10.1016/S0278-2391(99)90683-3

[13] Demir, Z., Velideglu, H., Sahin, U., Kurtay, A. and Coskunfirat, O.K. (2001) Preserved costal cartilage homograft application for the treatment of the tempromandibular joint ankylosis. Plastic and Reconstructive Surgery, 108, 44-51. doi:10.1097/00006534-200107000-00008

[14] Miyamoto, H., Kurita, K., Ogi, N., Ishimaru, J.I. and
Goss, A.N. (2000) The effect of an intraarticular bone fragment in the genesis of the temporomandibular joint ankylosis. International Journal of Oral and Maxillofacial Surgery, 29, 290-295. doi:10.1016/S0901-5027(00)80031-4

[15] Saeed, N.R. and Kent, J.N. (2003) A retrospective study of the costochondral graft in TMJ reconstruction. International Journal of Oral and Maxillofacial Surgery, 32, 606-609. doi:10.1054/ijom.2003.0418

[16] McCarthy, J.G., Schreiber, J., Karp, N., Thorne, C.H. and Grayson, B.H. (1992) Lengthening the human mandible by gradual distraction. Plastic \& Reconstructive Surgery, 89, 1-8. doi:10.1097/00006534-199201000-00001

[17] Molina, F. and Orfiz Monasterio, F. (1995) Mandibular elongation and remodeling by distraction: A farewell to major osteotomies. Plastic \& Reconstructive Surgery, 96, 825-840. doi:10.1097/00006534-199509001-00011

[18] Cohen, S.R. (1999) Craniofacial distraction with a modular internal distraction system: Evolution of design and surgical techniques. Plastic \& Reconstructive Surgery, 103, 1592-1607. doi:10.1097/00006534-199905060-00006

[19] Kaban, L.B., Moses, M.H. and Mulliken, J.B. (1988) Surgical correction of hemifacial microsomia in the growing child. Plastic \& Reconstructive Surgery, 82, 919. doi:10.1097/00006534-198882010-00003 